\title{
Compreendendo os significados das interações entre profissionais de saúde e idosos usuários de medicamentos
}

\section{Understanding the meanings of the interactions between health care professionals and elderly users of the medicine}

\section{Comprendendo los significados de las interaciones entre profesionales de la salud y los ancianos usuarios de medicamentos}

\author{
Divaldo Pereira de Lyra Júnior', Tatiane Cristina Marques", Adriana Inocenti Miasso'"', \\ Sílvia Helena De Bortoli Cassianilv
}

\section{RESUMO}

$O$ presente trabalho visa compreender e analisar os significados das interações entre profissionais de saúde (médicos e farmacêuticos) e idosos portadores de condições crônicas de saúde, na perspectiva dos usuários de medicamentos. $O$ estudo foi realizado em uma unidade de saúde do Município de Ribeirão Preto, em agosto de 2003. Esta análise secundária teve como referencial o Interacionismo Simbólico e os dados das entrevistas gravadas, no trabalho original, foram submetidos à análise temática de conteúdo. Os temas que emergiram demonstram que a ambivalência simbólica das interações permite diversas interpretações que vão desde o distanciamento e falta de confiança até a liberdade de expressão e o diálogo com o profissional de saúde. Compreender tais interações pode ser fundamental para melhorar - entendimento do processo saúde-doença, otimizar os cuidados à saúde e garantir o uso racional dos medicamentos.

Palavras chave: Idoso; Doença crônica; Uso de medicamentos; Pesquisa qualitativa.

\section{ABSTRACT}

The present work aims at understanding and analyzing the meanings of the interactions between health care professionals (doctors and pharmacists) and elderly with chronic heath conditions, in the perspective of the medicine users. The study was carried out a primary healthcare unit in Ribeirão Preto (SP), Brazil, in August 2003. This secondary analysis had as referencial the Symbolic Interacionism and the data of the recorded interviews, in the original work, had been submitted to the thematic analysis of content. The subjects that had emerged demonstrate that symbolic ambivalence of the interactions allows diverse interpretations that go since the distance and the lack of confidence until the liberty of speech and the dialogue with the health care professionals. Understanding such interactions can be basic to improve the agreement of the process health-illness, to optimize the cares to the health and to guarantee the rational use of medicines.

Key words: Aged; Chronic disease; Drug utilization; Qualitative research.

\section{RESUMEN}

El presente trabajo visa comprender y analizar los significados de las interaciones entre profesionales de la salud (médicos y farmacéuticos) y ancianos portadores de condiciones crónicas de salud, en la perspectiva de los usuarios de medicamentos. El estudio fue realizado en una unidad de atención primaria de salud en Ribeirão Preto (SP), Brasil, en agosto de 2003. Este análisis secundario tuvo como referencial el Interacionismo Simbólico y los datos de las entrevistas grabadas, en el trabajo original, fueron sometidos al análisis temático de contenido. Los temas que emergieron demuestran que la ambivalencia simbólica de las interaciones permite diversas interpretaciones que van desde el

\footnotetext{
Farmacêutico. Professor Doutor do Curso de Farmácia, Laboratório de Ensino e Pesquisa em Farmácia Social, Departamento de Fisiologia, Universidade Federal de Sergipe. E-mail: lyra_jr@hotmail.com

Farmacêutica. Mestranda do Departamento de Enfermagem Geral e Especializada da Escola de Enfermagem de Ribeirão Preto/ USP. E-mail: tatianecm@hotmail.com

III Enfermeira. Professora Doutora do Departamento de Enfermagem Psiquiátrica e Ciências Humanas da Escola de Enfermagem de Ribeirão Preto /USP. E-mail: amiasso@eerp.usp.br

IV Enfermeira. Professora Titular do Departamento de Enfermagem Geral e Especializada da Escola de Enfermagem de Ribeirão Preto /USP. E-mail:
} shbcassi@eerp.usp.br 
Lyra Júnior DP, Marques TC, Miasso AI, SHB Cassiani. Compreendendo os significados das interações entre profissionais de saúde e idosos usuários de medicamentos. Rev. Eletr. Enf. [Internet]. 2008; 10(3):591-9. Available from: http://www.fen.ufg.br/revista/v10/n3/v10n3a05.htm

distanciamiento y la falta de confianza hasta la libertad de expresión y el diálogo con el profesional de la salud. Comprender tal interación puede ser fundamental para mejorar el entendimiento del proceso salud-enfermedad,

\section{NTRODUÇÃO}

O envelhecimento da população mundial tem levado a maior prevalência das condições crônicas de saúde e, conseqüentemente, ao crescimento no consumo de medicamentos ${ }^{(1)}$. Essa multiplicidade de doenças e de medicamentos utilizados pelos idosos aumenta a dificuldade em compreender ou administrar prescrições ${ }^{(2-3)}$. Por isso, os índices de morbimortalidade relacionada aos medicamentos têm crescido, com impacto direto nos sistemas de saúde de todo o mundo ${ }^{(4)}$.

O uso de medicamentos impõe uma maior interação dos idosos com os familiares e profissionais de saúde. Nesse sentido, a literatura tem reconhecido a importância da equipe multiprofissional no cuidado aos idosos, pois os variados objetivos e abordagens com ação diferenciada corrigem a grande limitação das ações isoladas no tratamento, influenciando na efetivação da farmacoterapia e no controle das doenças ${ }^{(5-6)}$.

Diante do exposto, a comunicação pode ser uma ferramenta essencial para o cuidado na utilização dos medicamentos e na promoção da saúde, pois as práticas estão baseadas na interação com grande variedade de indivíduos, incluindo, pacientes, familiares e profissionais de saúde ${ }^{(7)}$. Para atender a essa nova demanda, estudos demonstram um incremento nas competências clínico-humanísticas, nas habilidades de comunicação e instrumentos utilizados por esses profissionais ${ }^{(8-9)}$.

Entretanto, ainda é fato a existência de difícil interação entre pacientes e profissionais de saúde, gerando consequências diretas nos seus cuidados, especialmente no uso dos medicamentos $^{(5,10-11)}$. Nesse contexto, a relevância desse estudo não se restringe apenas sobre como a pesquisa qualitativa poderá contribuir para implementação e consolidação das ações que visam o uso racional dos medicamentos no Município de Ribeirão Preto- optimizar los cuidados de la salud y garantizar el uso racional de los medicamentos.

Palabras clave: Anciano; Enfermedad crónica; Utilización de medicamentos; Investigación cualitativa.

SP, mas também como pode ser um instrumento efetivo de cunho social no que concerne a promoção da saúde.

Assim, partindo-se do pressuposto que em unidade de atenção primária à saúde ocorrem várias interações entre os idosos e os profissionais de saúde, utilizou-se para este estudo o referencial teórico denominado de interacionismo simbólico. Este referencial é originário da psicologia social e centrado na natureza da interação social, ou seja, nas atividades dinâmicas e sociais que acontecem entre e dentro de cada pessoa(12). Nesse referencial o significado é o eixo condutor do comportamento, definindo uma situação que precede uma ação. Nesse contexto, as partes que interagem "assumem o papel do outro", alinhando suas ações a de outros, direcionando o que estão fazendo ou o que vão fazer e atribuindo significados aos seus atos ${ }^{(13)}$.

A interação simbólica é, portanto, uma ferramenta teórica que possibilita a compreensão do fenômeno de uma maneira mais ampla, pois revela qual o significado que os idosos atribuem às relações com os profissionais de saúde, bem como, o que isso representa para o processo saúde-doença.

Embasado no contexto descrito, o presente estudo visa compreender e analisar os significados das interações entre os profissionais de saúde (médicos e farmacêuticos) e idosos portadores de condições crônicas de saúde, na perspectiva dos usuários de medicamentos.

\section{METODOLOGI A}

No presente estudo foi realizada a análise secundária dos dados obtidos em uma Tese de Doutorado intitulada "I mpacto de um programa de Atenção Farmacêutica nos resultados clínicos e humanísticos de um grupo de idosos assistidos na Unidade Distrital Básica de Saúde (UBDS) Dr. Ítalo Baruffi, Ribeirão Preto $(\mathrm{SP})^{\prime \prime(14)}$. A análise secundária é o uso de dados 
Lyra Júnior DP, Marques TC, Miasso AI, SHB Cassiani. Compreendendo os significados das interações entre profissionais de saúde e idosos usuários de medicamentos. Rev. Eletr. Enf. [Internet]. 2008; 10(3):591-9. Available from: http://www.fen.ufg.br/revista/v10/n3/v10n3a05.htm

reunidos em um estudo anterior para testar novas hipóteses ou abordar novas questões de pesquisa(15).

O banco de dados do estudo original contém informações sobre um grupo de 30 idosos, com idade entre 60 e 75 anos, de ambos os sexos, portadores de hipertensão arterial diagnosticada há pelo menos cinco anos, com seguimento ambulatorial realizado por médicos da referida UBDS e fazendo uso de medicamentos anti-hipertensivos padronizados pela Secretaria Municipal da Saúde de Ribeirão. A amostra foi determinada a partir da redução, delimitação e saturação teórica das categorias encontradas nos depoimentos, sendo que foram selecionados os 15 idosos cujo conteúdo das entrevistas apresentou maior profundidade quanto à interação com os profissionais de saúde (médicos e farmacêuticos). Desse modo, a saturação teórica foi alcançada quando os dados se tornaram repetitivos ou irrelevantes.

Durante esse estudo original, os idosos interagiram com seis profissionais de saúde (quatro médicos e dois farmacêuticos) vinculados à UBDS. O projeto original foi aprovado pelo Comitê de Ética em Pesquisa da Faculdade de Farmácia de Ribeirão Preto/USP (n.18/2003-CEP/FCFRP) e todos os idosos foram esclarecidos quanto às metas e a natureza das entrevistas, assinando um termo de consentimento pós-informação.

Ainda no estudo original, os idosos foram selecionados e individualmente entrevistados por um farmacêutico-pesquisador, de julho de 2003 a agosto de 2004, em uma sala da UBDS pertencente à Secretaria Municipal da Saúde de Ribeirão Preto (SP). Neste estudo foram utilizados apenas os dados referentes às duas primeiras entrevistas gravadas durante o estudo original, em agosto de 2003, com duração média de 60 minutos cada.

$\mathrm{Na}$ primeira entrevista foi aplicada um instrumento semi-estruturado, padronizado e previamente testado, que serviu para caracterizar os aspectos sócio-demográficos e econômicos dos idosos (gênero, idade, etnia, estado civil, ocupação, escolaridade, renda familiar e problemas de saúde), bem como, os medicamentos utilizados (prescritos ou não). Nas duas entrevistas também foram utilizados roteiros com questões abertas, dos quais foram obtidas as informações para análise dos dados.

No presente estudo foi utilizado o método de análise temática de conteúdo que é o conjunto de técnicas de análise das comunicações, buscando compreender os seus significados a partir de opiniões, atitudes, valores e crenças $^{(16)}$. Inicialmente, foi realizada uma leitura globalizada do material, e em seguida, as informações obtidas foram identificadas e analisadas; confirmadas ou refutadas a partir dos pressupostos da pesquisa qualitativa e da ampliação do conhecimento sobre o tema estudado, considerando o contexto em que o mesmo está inserido(16). A análise das entrevistas gerou duas categorias temáticas que serão apresentadas e discutidas a seguir.

\section{RESULTADOS E DISCUSSÃO}

\section{Caracterização dos informantes}

Dos 15 informantes, dez eram do sexo feminino e cinco masculino, sendo oito brancos e sete negros. A idade média do grupo foi de 67 anos, sendo a maioria (dez) casada e com uma renda familiar média de três salários mínimos (US\$ 400). As variáveis clínicas e sóciodemográficas do grupo em estudo foram apresentadas no Quadro 1: 
Lyra Júnior DP, Marques TC, Miasso AI, SHB Cassiani. Compreendendo os significados das interações entre profissionais de saúde e idosos usuários de medicamentos. Rev. Eletr. Enf. [Internet]. 2008; 10(3):591-9. Available from: http://www.fen.ufg. br/revista/v10/n3/v10n3a05.htm

Quadro 1: Variáveis clínicas e sócio-demográficas dos idosos. Ribeirão Preto-SP, 2003.

\begin{tabular}{|c|c|}
\hline Iniciais & Características sócio-demográficas \\
\hline LMA & $\begin{array}{l}\text { q } 62 \text { anos, branca, casada, aposentada, com ensino fundamental incompleto, apresentou } \\
\text { cinco condições crônicas de saúde }\end{array}$ \\
\hline PMB & $\begin{array}{l}\text { + } 69 \text { anos, branca, casada, dona de casa, cursou ensino médio, apresentou quatro } \\
\text { problemas de saúde, sendo duas condições crônicas }\end{array}$ \\
\hline ABC & $\begin{array}{l}\text { o } 71 \text { anos, negro, casado, aposentado, com ensino fundamental incompleto, apresentou três } \\
\text { condições crônicas de saúde }\end{array}$ \\
\hline AAFM & $\begin{array}{l}\text { + } 75 \text { anos, negra, viúva, dona de casa, cursou ensino médio, apresentou três condições } \\
\text { crônicas de saúde }\end{array}$ \\
\hline MMRM & $\begin{array}{l}\text { + } 62 \text { anos, branca, viúva, auxiliar de serviços gerais, com ensino médio incompleto, } \\
\text { apresentou oito problemas de saúde, sendo três condições crônicas }\end{array}$ \\
\hline ISO & $\begin{array}{l}\text { + } 71 \text { anos, negra, viúva, pensionista, com ensino fundamental incompleto, apresentou sete } \\
\text { problemas de saúde, sendo três condições crônicas }\end{array}$ \\
\hline AFC & $\begin{array}{l}\text { q } 63 \text { anos, branca, casada, aposentada, com ensino fundamental incompleto, apresentou } \\
\text { cinco problemas saúde }\end{array}$ \\
\hline I BP & $\begin{array}{l}\text { o } 61 \text { anos, negro, solteiro, pintor, com ensino fundamental incompleto, apresentou duas } \\
\text { condições crônicas de saúde }\end{array}$ \\
\hline GS & $\begin{array}{l}\text { + } 75 \text { anos, negra, solteira, aposentada, com ensino fundamental incompleto, apresentou dez } \\
\text { problemas de saúde, sendo quatro condições crônicas }\end{array}$ \\
\hline MCR & $\begin{array}{l}\text { N } 60 \text { anos, branco, casado, aposentado, com ensino fundamental incompleto, apresentou } \\
\text { três problemas de saúde, sendo duas condições crônicas }\end{array}$ \\
\hline PAP & $\begin{array}{l}\text { o } 61 \text { anos, negro, casado, pedreiro, com ensino fundamental incompleto, apresentou três } \\
\text { problemas de saúde, sendo duas condições crônicas }\end{array}$ \\
\hline J CM & $\begin{array}{l}\text { N } 68 \text { anos, branco, casado, aposentado, cursou ensino fundamental completo, apresentou } \\
\text { duas condições crônicas de saúde }\end{array}$ \\
\hline MST & $\begin{array}{l}\text { + } 75 \text { anos, branca, casada, dona de casa, com ensino fundamental incompleto, apresentou } \\
\text { seis problemas de saúde, sendo três condições crônicas }\end{array}$ \\
\hline I PC & $\begin{array}{l}\text { q } 66 \text { anos, negra, casada, dona de casa, com ensino fundamental incompleto, apresentou } \\
\text { duas condições crônicas de saúde }\end{array}$ \\
\hline DMG & $\begin{array}{l}\text { q } 65 \text { anos, branca, casada, dona de casa, com ensino fundamental incompleto, apresentou } \\
\text { quatro problemas de saúde, sendo duas condições crônicas }\end{array}$ \\
\hline
\end{tabular}

A partir dos dados das entrevistas foram formulados dois temas que agruparam as interações estabelecidas entre os profissionais de saúde (médicos e farmacêuticos) e os idosos portadores de condições crônicas de saúde, na perspectiva dos usuários de medicamentos: 1 "Às vezes eu não faço o que ele manda": dificuldades na comunicação com os profissionais de saúde; 2 - "Ele é atencioso": valorizando os cuidados prestados pelos profissionais de saúde. Os temas que emergiram mostram a ambivalência do processo de interação entre estes profissionais de saúde e pacientes, a partir das falas dos idosos, a seguir:

Tema 1: "Às vezes eu não faço o que ele manda": dificuldades na comunicação com os profissionais de saúde

As expectativas do paciente variam de acordo com o seu nível sócio-econômicocultural, o acesso aos serviços de saúde, os anseios da família e da comunidade em que vive, o nível de dependência de terceiros para locomoção, custo e financiamento do tratamento, o nível de desconforto frente aos sintomas, o nível cognitivo, entre outros. Logo, os profissionais deveriam buscar estabelecer uma interação com os idosos que visasse atender a esses anseios. Todavia, o processo de mercantilização da saúde e de seus cuidados, a formação dos médicos e farmacêuticos, baseada no paradigma tecnicista, e a queda na qualidade dos cuidados, gerou carências na maioria dos atendimentos, penalizando os usuários na relação com os profissionais e levando à falta de confiança, principalmente entre os idosos, conforme se pode observar nos depoimentos abaixo:

Às vezes eu não faço o que o médico manda. Sei lá, não confio... (IBP).

A gente desconfia das explicações do médico. Ele é novinho, no dia que eu falei pra ele que eu tava sentindo mal, ele se sentiu mais mal do que eu (GS). 
Lyra Júnior DP, Marques TC, Miasso AI, SHB Cassiani. Compreendendo os significados das interações entre profissionais de saúde e idosos usuários de medicamentos. Rev. Eletr. Enf. [Internet]. 2008; 10(3):591-9. Available from: http://www.fen.ufg.br/revista/v10/n3/v10n3a05.htm

Tem umas coisas que eles diz que não sei não, parece que nem eles têm certeza se o remédio funciona (MCR).

Tem uns conselhos que o médico está me dando sobre os medicamentos que eu posso até achar que está certo... (AAFM).

Eu acho que se médico receitou esse remédio fraquinho, ele deve achar que está fazendo certo?! (J CM).

O sofrimento humano, as percepções de dor ou de prazer no corpo para serem humanizadas precisam tanto que as palavras com que 0 sujeito as expressa sejam reconhecidas pelo "outro", quanto esse sujeito precisa escutar e reconhecer palavras do "outro" (11,17). Deste modo, humanizar é a compreensão recíproca das palavras, nos seus âmbitos concreto e intersubjetivo, garantindo a sua dignidade ética. Os relatos dos idosos, porém, mostraram que os médicos e farmacêuticos pareciam desconsiderar tais aspectos:

Falar dos remédios o médico falou, mas não deu para entender... Ele fala daquele jeito... difícil demais para mim... (AFC)".

Tem médico que anda correndo por aqui que às vezes nem ouve o paciente direito, mas passa logo um remédio, né?! (AAFM ).

Aquele lá (o médico), nem escuta o que a gente pergunta! (LMA).

A linguagem é responsável pela descoberta de meios pessoais de comunicação com o "outro", sem que ocorra uma desumanização recíproca ${ }^{(17)}$. Todavia, a desumanização se caracteriza por desequilíbrio de poder, em que a linguagem delimita o lado dominante do dominado. Nessas interações assimétricas e verticalizadas, o dominante desconsidera o "outro", reduzindo-o a situação de objeto que pode ser manipulado em função dos interesses e necessidades do primeiro. No estudo, o cenário atual de desumanização do serviço de saúde apareceu em alguns depoimentos, ressaltando principalmente 0 distanciamento e desinteresse da maioria dos profissionais de saúde com os idosos:
Dessa vez o médico nem olhou, foi passando logo o remédio (IPC).

Eu vou ao consultório desses médicos e falo... Eu tenho gastrite! Eles não ligam, deixam pra lá! (AAFM).

Diante desse quadro, a maioria dos médicos não considerou os déficits cognitivos e nem buscou referências psico-sociais para orientar os idosos, empregando exclusivamente o modelo tecnicista aprendido ${ }^{(10,18)}$. Seguindo esta lógica, os profissionais de saúde estabeleceram relações de dominação caracterizadas pela grafia ilegível e pela linguagem técnico-científica inacessível à compreensão dos idosos:

Não dá para entender nada... letra de médico, né?! (ISO).

Lê a receita, a gente tenta, mas entender que é bom... (AAFM).

Falar dos remédios o médico falou, mas não deu para entender... Ele fala muito difícil (AFC).

Entendo pouco... Ele (o farmacêutico) fala igual ao médico (IPC).

De acordo com a literatura, a maioria dos médicos não considera os déficits cognitivos e nem busca referências psico-sociais para orientar os idosos, empregando exclusivamente o modelo tecnicista aprendido ${ }^{(10,18)}$.

No processo de medicalização, as ações dominantes dos profissionais de saúde estão intimamente ligadas à onipotência e à hierarquização do saber científico sobre o empírico, enquanto que os idosos, doentes, consomem os medicamentos ordenados sem entender para que serve, como usá-los e que cuidados adotar para minimizar ou prevenir potenciais riscos a sua saúde. No estudo, fatores como a baixa escolaridade e os déficits fisiológico-cognitivos característicos dessa faixa etária dificultaram 0 entendimento das orientações ministradas pelos profissionais de saúde, segundo os depoimentos:

É difícil, tem muitos remédios e nós misturamos todas as explicações na cabeça (DMG).

O médico explicou rápido demais e nem deu pra ouvir direito $(A B C)$. 
Lyra Júnior DP, Marques TC, Miasso AI, SHB Cassiani. Compreendendo os significados das interações entre profissionais de saúde e idosos usuários de medicamentos. Rev. Eletr. Enf. [Internet]. 2008; 10(3):591-9. Available from: http://www.fen.ufg. br/revista/v10/n3/v10n3a05.htm

Os farmacêuticos explicam direitinho, mas podiam explicar melhor... a cabeça não é a mesma, nós somos idosos, né? (MST).

Em geral, a figura mítica dos médicos (ou dos profissionais de saúde), a idealização e a supervalorização constrangem e por vezes inibem o idoso. Segundo Martins ${ }^{(18)}$, quase sempre a relação é a do que sabe, do que manda, do que tem poder, e daquele que não sabe, que obedece, o oprimido. Tal significado foi observado nas falas, sob a forma de receio de confronto e, sobretudo, de passividade:

Tenho dúvidas, mas eu tenho assim... receio de falar... porque ele é médico, né?! (GS).

... se o médico receitou esse, vamos tomar esse (J CM).

O medicamento não funciona, mas eu não comento com ele (médico)... porque eu não gosto de me meter, né?! (J CM).

Nas relações em que o paradigma tecnicista ainda predomina a consulta é ameaçadora para os idosos ${ }^{(10)}$. Segundo a literatura só é possível humanizar a partir de nossa própria humanização(19). Por isso, o profissional de saúde deve compreender que as relações dominadoras de poder são prejudiciais à comunicação com os idosos.

Nesse contexto, sem comunicação não há humanização e esta dependerá da capacidade do profissional de saúde de falar e escutar, de forma horizontalizada, pois as coisas do mundo só se tornam humanas quando passam pelo diálogo com nossos semelhantes ${ }^{(17)}$. Assim, foi possível observar no próximo tema como os profissionais de saúde que estabeleceram o diálogo foram vistos por alguns idosos usuários da UBDS.

\section{Tema 2: "Ele é atencioso": valorizando os cuidados prestados pelos profissionais de saúde}

As relações dialógicas se fundamentam na empatia. A empatia deixa "rolar os sentimentos", como uma forma de sentir ou tentar sentir o que o "outro" sente, pois nessa troca de emoções e de preocupações surgem maiores oportunidades de encontrar alternativas viáveis e adequadas para se cuidar do idoso ${ }^{(14,20)}$. Logo, essas interações devem ser norteadas por princípios, como: ética, respeito mútuo, confidencialidade e, sobretudo, coresponsabilidade.

O cuidar envolve verdadeiramente uma ação interativa, calcada nos valores e nos conhecimentos do ser que cuida "para" e "com" o ser que é cuidado ${ }^{(18)}$. Nesse contexto, o cuidar pode ser entendido como a experiência de atender ao chamado do "outro", sendo o "outro" o idoso que é vulnerável, demanda atenção e responsabilidade profissional ${ }^{(11)}$. Nos depoimentos de alguns idosos foram enfatizados aspectos positivos das orientações feitas por alguns profissionais de saúde, como a atenção e o respeito:

Eu acho o médico muito bom, porque é muito atencioso e respeita nós... (AAFM).

Acho o médico muito atencioso com a gente, sabe?! Ele ouve a gente direitinho (AAFM).

Ele presta atenção. Tem dia que nem precisa falar... O farmacêutico já sabe que não estou bem (GS).

A farmacêutica orienta sempre. Ela é muito atenciosa (MFC).

A humanização é uma ferramenta que visa tornar o cuidado aos pacientes efetivo e diferenciado, considerando cada ser bio-psicosocial como único. Ao mesmo tempo, faz contraposição ao atual modelo de cuidado tecnicista, mecânico e "desprovido de sentimentos"(19). Nesse sentido, as interações entre os profissionais de saúde e os idosos não podem ser mais "desprovidas de sentimentos", mas fundamentadas em um processo que permite deixar "rolar os sentimentos"(20). Tais sentimentos são refletidos no fato dos idosos ressaltarem estar felizes por serem respeitados e reconhecidos como gente, como citado:

O médico é uma pessoa doce, generoso mesmo... Ele trata a gente feito gente, respeita mesmo ( $A B C)$.

O médico é um ser humano mesmo, respeitoso e preocupado com a saúde da gente... Quem não fica feliz de ser tratado feito gente?! (AFC). 
Lyra Júnior DP, Marques TC, Miasso AI, SHB Cassiani. Compreendendo os significados das interações entre profissionais de saúde e idosos usuários de medicamentos. Rev. Eletr. Enf. [Internet]. 2008; 10(3):591-9. Available from: http://www.fen.ufg. br/revista/v10/n3/v10n3a05.htm

O farmacêutico é educado e a gente sente que ele tem respeito e se interessa de verdade em cuidar de nós (MST).

Quando o profissional de saúde compreende que o processo de troca, de sentir ou tentar sentir o que o "outro" está sentido, é benéfico para o idoso, passa a usar esta interação como um relevante instrumento de trabalho ${ }^{(14)}$. Nessa perspectiva, o diálogo mediou o estabelecimento de interações horizontalizadas e simétricas entre idosos e profissionais de saúde, alicerçadas no cuidado, na confiança e na liberdade de expressão:

O médico é um desses que não se encontra mais... cuidadoso, explica com calma e se preocupa se a gente entendeu (MST).

Ele (o farmacêutico) é cuidadoso, fala as palavras certas, do jeito que nós fala... Assim é mais fácil de entender! (JCM).

Tenho muito assunto com o médico... Ele me deixa a vontade para perguntar e por isso, confio muito! (PMB).

Eu tenho liberdade com esse médico, ele é muito cuidadoso... é de confiança da gente... (IPC).

Tais interações possibilitaram aos idosos agregarem informações sanitárias em sua estrutura cognitiva que permitiram aprimorar e redimensionar a sua relação com o processo saúde-doença, seus medicamentos e os profissionais que cuidam da sua saúde.

O diálogo aberto e franco é fundamental para consolidar a interação entre profissionais e idosos, pois busca diminuir a distância existente entre os mesmos, melhorando o entendimento sobre as doenças e reduzindo os riscos relacionados ao uso dos medicamentos. No presente estudo foi possível perceber que os profissionais que apoiaram a sua prática no diálogo, foram reconhecidos e valorizados pelos idosos, conforme os depoimentos:

Eu acho ele muito bom! Eu ouço tanto o povo falar mal dos médicos do SUS... Eu acho que pra mim ele é um médico do SUS, bom! (AAFM).
Eu acho que estou bem servida de médicos (ISO).

Esse médico foi muito bom, foi bem caprichoso (GS).

Sabe o que é, é que eu valorizo os bons médicos (PMB).

Esse farmacêutico é muito bom, com ele eu tenho mais liberdade que com o médico... Ele cuida de mim! (JCM).

Ao refletir sobre o significado do cuidado humanizado, tendo a cultura como um sistema de símbolos pelos quais os seres humanos percebem o mundo que habitam, aprendendo a viver nele, os profissionais de saúde têm a responsabilidade e o compromisso ético de resgatar o sentido das suas ações, e isso só será possível a partir da conscientização de que o ser humano é capaz de buscar a si mesmo, a sua essência e, por conseqüência, o “outro"(19).

Aspectos relativos à percepção dos idosos quanto à interação com outros profissionais de saúde que não fossem médicos e farmacêuticos também não foram abordados, podendo ser objeto de novos estudos.

\section{CONSI DERAÇÕES FI NAIS}

A pesquisa qualitativa à luz das ciências sociais traz elementos relevantes, capazes de responder a questões específicas que não podem ser quantificadas, como os significados, crenças, valores e atitudes. Assim, o presente estudo mostrou que, na perspectiva dos idosos, as principais dificuldades de interação com médicos e farmacêuticos estão ligadas às questões comportamentais e de conhecimento, interferindo direta ou indiretamente no uso dos medicamentos.

$\mathrm{Na}$ relação com os profissionais de saúde que fundamentaram o seu atendimento no paradigma tecnicista, os idosos atribuíram significados negativos como distanciamento, desinteresse e em conseqüência, falta de confiança. Nos depoimentos do grupo em estudo também ficaram explícitos significados como a falta de informação, a passividade e o receio de confronto com os profissionais.

Em contraposição, os profissionais de saúde que se dedicaram a atender o "outro" foram percebidos como respeitosos, atenciosos, 
Lyra Júnior DP, Marques TC, Miasso AI, SHB Cassiani. Compreendendo os significados das interações entre profissionais de saúde e idosos usuários de medicamentos. Rev. Eletr. Enf. [Internet]. 2008; 10(3):591-9. Available from: http://www.fen.ufg.br/revista/v10/n3/v10n3a05.htm

preocupados e confiáveis. Essas interações horizontalizadas e simétricas foram construídas nos pilares sólidos do respeito mútuo, fruto do reconhecimento do idoso como ser humano igual ao profissional de saúde, e do diálogo, que proporcionou a liberdade para expressar suas dúvidas, medos e preocupações.

Os significados encontrados levam ao entendimento de que as relações entre profissionais de saúde e idosos usuários de medicamentos se estabelecem alicerçadas na interação social, ou seja, que se interpretam uns aos outros. Compreender tais interações pode ser fundamental para melhorar o entendimento do processo saúde-doença, otimizar os cuidados à saúde do idoso e garantir o uso correto e seguro dos medicamentos.

Agradecimentos: Este trabalho contou com o suporte financeiro da Fundação de Amparo à Pesquisa do Estado de São Paulo (FAPESP) e da Coordenação de Aperfeiçoamento de Pessoal de Nível Superior (CAPES). A Camila Agustoni, Daniela Odnicki, Giovana Martins e Mayra Menegheti pela importante colaboração no início deste estudo. Aos funcionários, médicos e farmacêuticos da UBDS Dr. Ítalo Baruffi e, em especial, aos todos os informantes que contribuíram na realização deste trabalho.

\section{REFERÊNCI AS}

1. Fanhani HR, Takemura OS, Cuman RKN, Seixas FAV, Andrade OG. Consumo de medicamentos por idosos atendidos em um centro de convivência no noroeste do Paraná, Brasil. Rev Bras Geriatr Gerontol. 2007; 10(3).

2. Lyra Junior DP, Prado MCTA, Abriata JP, Pelá IR. Recetas médicas como causantes de riesgo de problemas relacionados con medicamentos. Seguimiento Farmacoterapêutico. 2004;2(2): 86-96.

3. Rocha $\mathrm{CH}$, Oliveira APS, Ferreira C, Faggiani FT, Schroeter G, Souza ACA, DeCarli GA, Morrone FB, Werlang.MC. Adesão à prescrição médica em idosos de Porto Alegre, RS. Ciência e Saúde Coletiva 2008 abr; 13(Suppl): 703-10.

4. Llimós FF, Faus MJ . Importance of medicinerelated problems as risk factors. The Lancet. 2003; 362(9391): 1239.
5. Lyra Junior DP, Amaral RT, Veiga EV, Cárnio EC, Nogueira MS, Pelá IR. A farmacoterapia no idoso: uma revisão sobre a abordagem multiprofissional no controle da hipertensão arterial sistêmica. Revista Latino-Americana de Enfermagem. 2006; 14(3): 435-42.

6. Rodrigues RAP, Kusumota L, Marques S, Fabrício SCC, Cruz IR, Lange C. Política nacional de atenção ao idoso e a contribuição da enfermagem. Texto Contexto Enferm. 2007; 16(3): 536-45.

7. Berger BA. Communication skills for pharmacists: building relationships, improving patient care. 2nd edition. Washington: APHA; 2002.

8. Miasso Al. "Entre a cruz e a espada": o significado da terapêutica medicamentosa para a pessoa com transtorno afetivo bipolar, em sua perspectiva e na de seu familiar. [tese]. [Ribeirão Preto]: Escola de Enfermagem de Ribeirão Preto/USP; 2006.

9. Beardsley RS. Communication skills development in colleges of pharmacy. Am J Pharm Education. 2001;65(4): 307-14.

10. Grosseman S, Patrício ZM. Relação médicopaciente e o cuidado humano subsídios para promoção da educação médica. Revista Brasileira de Educação Médica. 2004;28(2):99105.

11. Oliveira DR, Shoemaker SJ. Achieving patient centeredness in pharmacy practice: openness and the pharmacist's natural attitude. J Am Pharm Assoc. 2006; 46(1): 56-66.

12. Blumer $\mathrm{H}$. Symbolic interactionism: perspective and method. Berkeley: University of California; 1969.

13. Haguette TMF. Metodologias qualitativas na sociologia. Petrópolis: Vozes; 1999.

14. Lyra Junior DP. Impacto de um programa de Atenção Farmacêutica, no cuidado de um grupo de idosos atendidos na Unidade Básica Distrital de Saúde Dr. Ítalo Baruffi, Ribeirão Preto (SP) [tese].[Ribeirão Preto]: Faculdade de Ciências Farmacêuticas de Ribeirão Preto/USP; 2005.

15. Polit DF, Beck CT, Hungler BP. Fundamentos de Pesquisa em Enfermagem: métodos, avaliação e utilização. $5 a$ ed. Porto Alegre: Artes Médicas; 2004.

16. Bardin, L. Análise de conteúdo. 3ạ edição. Lisboa: Edições 70, 2004. 
Lyra Júnior DP, Marques TC, Miasso AI, SHB Cassiani. Compreendendo os significados das interações entre profissionais de saúde e idosos usuários de medicamentos. Rev. Eletr. Enf. [Internet]. 2008; 10(3):591-9. Available from: http://www.fen.ufg. br/revista/v10/n3/v10n3a05.htm

17. Betts J. Considerações sobre o que é humanizar. [Internet] [cited 2003 out 9] Available from: http://www.portalhumaniza.org.br/ph/texto.asp ?id=37.

18. Martins DG. Relação médico - paciente em hospital público: implicações psicológicas quanto a variável obesidade. Psicologia: Teoria e Prática. 1999; 1(2):80-9.

19. Vila VSC, Rossi LA. O significado cultural do cuidado humanizado em unidade de terapia intensiva: muito falado e pouco vivido. Revista Latino-Americana de Enfermagem. 2002; 10(2): 137-44.

20. Lyra Junior DP, Amaral RT, Abriata JP, Pelá IR. Satisfacción como resultado de un programa de atención farmacéutica para pacientes ancianos en Ribeirão Preto - São Paulo (Brasil). Seguimiento Farmacoterapêutico6. 2005;3(1):30-42.

Artigo recebido em 21.12.07

Aprovado para publicação em 30.09.08 